Citation: I. Ancillotti (2021) Pier Cesare Rivoltella, Tempi della lettura. Media, pensiero, accelerazione. Brescia: Morcelliana (2020). Media Education 12(2): 99-100. doi: 10.36253/me-12226

Received: October, 2021

Accepted: November, 2021

Published: December, 2021

Copyright: (c) $2021 \mathrm{I}$. Ancillotti. This is an open access, peer-reviewed article published by Firenze University Press (http://www.fupress.com/me) and distributed under the terms of the Creative Commons Attribution License, which permits unrestricted use, distribution, and reproduction in any medium, provided the original author and source are credited.

Data Availability Statement: All relevant data are within the paper and its Supporting Information files.

Competing Interests: The Author(s) declare(s) no conflict of interest.
Recensioni

\section{Pier Cesare Rivoltella, Tempi della lettura. Media, pensiero, accelerazione. Brescia: Morcelliana (2020)}

\author{
Ilaria AnCillotti \\ Università di Firenze \\ ilaria.ancillotti@unifi.it
}

Se già da più di un decennio si può parlare di vite "onlife" (Floridi, 2015) - contemporaneamente nel virtuale e nella realtà -, con la pandemia di Covid-19 scoppiata ad inizio 2020 l'approccio di una persona media alle tecnologie e al digitale si è indubbiamente intensificato. Oggi, potremmo definire l'intera società "onlife": una società in cui si può facilmente lavorare da casa in modalità smart e in cui è possibile trovare classi scolastiche con lezioni svolte in modalità blended. Naturalmente, la pervasività del digitale nelle attività sociali ha alimentato anche il dibattito tra educazione/apprendimento e digitale, facendo emergere nuovi spunti di riflessione.

Nel volume Tempi della lettura. Media, pensiero, accelerazione, Pier Cesare Rivoltella, docente di Tecnologie dell'istruzione e dell'apprendimento presso la Facoltà di Scienze della Formazione dell'Università Cattolica di Milano e direttore del CREMIT, affronta uno dei temi più scottanti dell'attuale panorama educativo: il rapporto tra letto-scrittura, tempo e digitale.

Quanta influenza ha la componente 'tempo' sul processo di letto-scrittura? È stato l'avvento del digitale a causare le difficoltà di lettura e di comprensione del testo delle nuove generazioni?

In contrasto a quanto si potrebbe pensare, oggi si legge quantitativamente di più rispetto al passato ma qualitativamente peggio: leggiamo continuamente e-mail, messaggi ed articoli con una media di tempo di lettura di pochi secondi.

Sembrerebbe che la "lettura profonda" (p. 17) stia lasciando il campo a quella veloce, con grosse conseguenze sul piano della comprensione del testo.

Nella prima parte del volume Rivoltella parte da questa constatazione, riflettendo poi sulle possibili cause, a partire da quella che sembrerebbe la più evidente: l'influenza dei media digitali.

La lettura e la scrittura sono significativamente cambiate da quando i media digitali sono diventati parte integrante della quotidianità. La lettura è diventata smart: con le tecnologie mobili si può leggere costantemente e ovunque, facilitati da "contenuti pacchettizzati" (p. 13) e letture sintetiche. La scrittura è diventata modificabile ed ipertestuale, ma anche breve e concisa. 
Eppure, l'autore ci fa osservare che non sono i media in sé ad aver compromesso i tempi di lettura, di scrittura e di pensiero; bensì, essi sono figli del loro tempo e in quanto tali sono stati creati per assecondare la "società dell'accelerazione" (p. 10).

Il punto cruciale della questione è che il tempo nella società odierna si è ridotto all'istante, portando l'individuo a dedicare sempre meno tempo ad attività slow come la lettura. Un problema, dunque, culturale che ha richiesto una "nuova economia dell'attenzione e del pensiero" (p. 82).

Il capitolo terzo, attraverso riferimenti alle neuroscienze, chiarisce cosa intende l'autore per "nuova economia dell'attenzione e del pensiero". L'attenzione da focalizzata è diventata per lo più distribuita, capace di spostarsi velocemente da uno stimolo all'altro, per rispondere alle molteplici richieste del quotidiano; il pensiero è diventato veloce, segue il suo stream of consciousness, è altamente intuitivo e competente sul problem solving.

Nonostante il capitolo affronti una materia piuttosto complessa, la trattazione accompagnata da alcune immagini riesce ad illustrare chiaramente i cambiamenti che hanno coinvolto alcune funzioni cerebrali e perché, anche ad un fruitore privo di conoscenze neuroscientifiche.

Nei capitoli finali, Rivoltella sviluppa una riflessione critica su alcune possibili soluzioni a questa fugacità del tempo, recuperando le considerazioni filosofiche illustrate nelle parti iniziali del volume. Rispetto al problema della mancanza di tempo - per apprendere, per leggere, per vivere - c'è chi propone un "approccio accelerazionista", puntando sull'evoluzione della tecnologia fino alla completa sostituzione del lavoro umano (es. robot) e c'è chi prospetta un "approccio decelerazionista", suggerendo di entrare di nuovo intimamente in contatto con il mondo e con il proprio spirito, riprendendo i propri spazi (per qualche giorno o per sempre).

Tuttavia, entrambe le soluzioni tamponano soltanto un vasto problema.

Dunque, che fare?

Il libro si chiude con un potente messaggio dell'autore: Age quod agis, fa bene quel che fai. Per il professore, l'unica soluzione praticabile è l'“etica del presente" (p. 126), concentrarsi su quel che si sta facendo e farlo bene. 